\title{
Non-classical logic and undecidability
}

\author{
Open Mathematics Collaboration*†
}

July 22,2020

\begin{abstract}
We elaborate a conjecture by applying the definition of Non-Axiomatic System [1] in Non-Classical Logic.
\end{abstract}

keywords: non-classical logic, undecidable proposition, non-axiomatic system

The most updated version of this paper is available at https://osf.io/h4f7p/download

\section{Introduction}

1. Non-classical logic extends classical logic [2].

*All authors with their affiliations appear at the end of this paper.

†Corresponding author: mplobo@uft.edu.br | Join the Open Mathematics Collaboration 


\section{Non-classical proposition}

2. This sentence is false.

3. (2) can be expressed mathematically by $P \equiv \neg P$.

4. $P$ is neither true nor false.

5. Let the truth value of neither true nor false be denoted by $i$, i.e., $\delta(P)=i$.

6. The truth value of a vacuously true sentence $\mathcal{V}_{t}$ is $\bar{\delta}\left(\mathcal{V}_{t}\right)=i[3]$.

7. Let $\mathcal{N C}=\left\{F_{i}: i \in \mathbb{N}\right\}$, where $F_{i}$ are mathematical formulas such that $\bar{\delta}\left(F_{i}\right) \neq 0$ and $\bar{\delta}\left(F_{i}\right) \neq 1$.

\section{Definitions}

8. $D=$ undecidable theorem

9. NAS = Non-Axiomatic System [1]

10. $\mathcal{N N C}=\mathcal{N C} \cap \mathcal{M}$

\section{Conjecture}

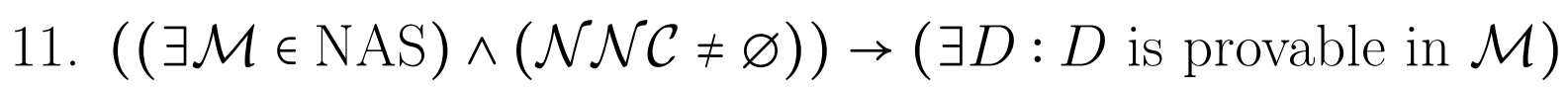

\section{Open Invitation}

Review, add content, and co-author this paper $[4,5]$. Join the Open Mathematics Collaboration (https://bit.ly/ojmp-slack). Send your contribution to mplobo@uft.edu.br. 


\section{Open Science}

The latex file for this paper together with other supplementary files are available [6].

\section{Ethical conduct of research}

This original work was pre-registered under the OSF Preprints [7], please cite it accordingly [8]. This will ensure that researches are conducted with integrity and intellectual honesty at all times and by all means.

\section{Acknowledgement}

+ Center for Open Science

https://www.cos.io

+ Open Science Framework

https://osf.io

\section{References}

[1] Lobo, Matheus P. "Mathematics Beyond Axioms." OSF Preprints, 5 Sept. 2019. https://doi.org/10.31219/osf.io/adrjq

[2] Priest, Graham. An introduction to non-classical logic: From if to is. Cambridge University Press, 2008.

[3] Lobo, Matheus P. "Vacuously True Sentences: Are They Non-boolean and Undecidable?." OSF Preprints, 26 July 2019. https://doi.org/10.31219/osf.io/8r3j2

[4] Lobo, Matheus P. "Microarticles." OSF Preprints, 28 Oct. 2019. https://doi.org/10.31219/osf.io/ejrct 
[5] Lobo, Matheus P. "Simple Guidelines for Authors: Open Journal of Mathematics and Physics." OSF Preprints, 15 Nov. 2019. https://doi.org/10.31219/osf.io/fk836

[6] Lobo, Matheus P. "Open Journal of Mathematics and Physics (OJMP)." OSF, 21 Apr. 2020. https://doi.org/10.17605/osf.io/6hzyp

[7] COS. Open Science Framework. https://osf.io

[8] Lobo, Matheus P. "Non-classical Logic and Undecidability." OSF Preprints, 21 June 2020. https://doi.org/10.31219/osf.io/h4f7p

\section{The Open Mathematics Collaboration}

Matheus Pereira Lobo (lead author, mplobo@uft.edu.br) $)^{1,2}$ https://orcid.org/0000-0003-4554-1372

${ }^{1}$ Federal University of Tocantins (Brazil)

${ }^{2}$ Universidade Aberta (UAb, Portugal) 